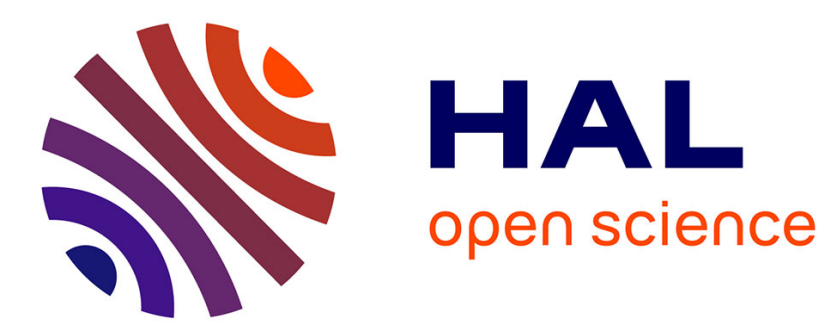

\title{
Copper Catalyzed Decarboxylative Functionalization of Ketoacids
}

\author{
Adrien Quintard
}

\section{To cite this version:}

Adrien Quintard. Copper Catalyzed Decarboxylative Functionalization of Ketoacids. Chemical Record, In press, 10.1002/tcr.202100045 . hal-03366964

\section{HAL Id: hal-03366964 \\ https://hal.science/hal-03366964}

Submitted on 5 Oct 2021

HAL is a multi-disciplinary open access archive for the deposit and dissemination of scientific research documents, whether they are published or not. The documents may come from teaching and research institutions in France or abroad, or from public or private research centers.
L'archive ouverte pluridisciplinaire HAL, est destinée au dépôt et à la diffusion de documents scientifiques de niveau recherche, publiés ou non, émanant des établissements d'enseignement et de recherche français ou étrangers, des laboratoires publics ou privés. 
Copper Catalyzed Decarboxylative Functionalization of Ketoacids

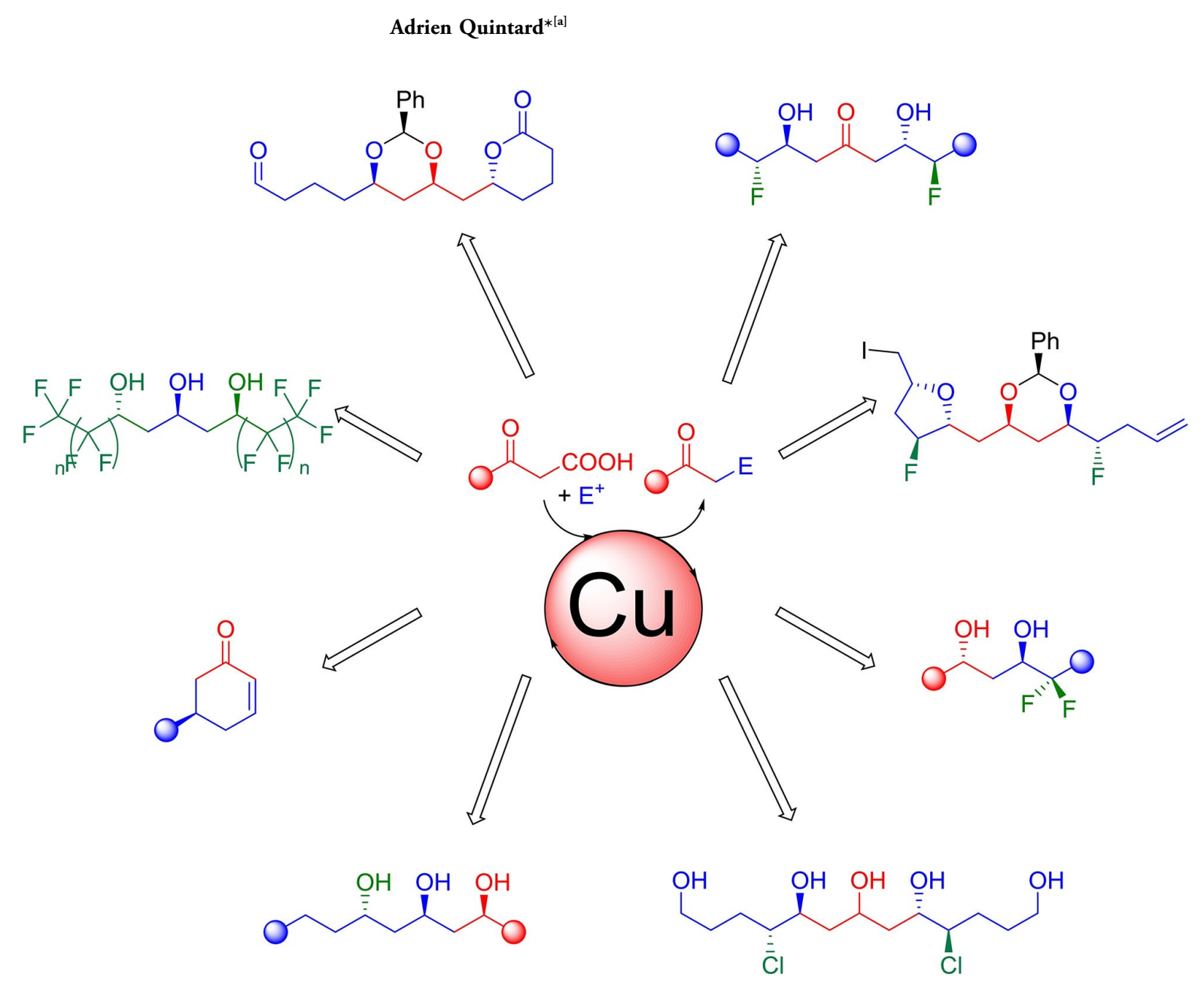




\begin{abstract}
Selective copper catalyzed activation of ketoacids and notably bio-sourced 1,3acetonedicarboxylic acid, represents an attractive strategy to solve key synthetic challenges. Condensation with aldehydes under exceedingly mild conditions can create more rapidly known natural products scaffolds such as 1,3 polyols. In this account, the recent progress in this field, notably through multicatalytic combination with organocatalysis is described. In addition to the rapid preparation of natural product fragments, cascade incorporation of fluorine also provided new type of synthetic analogues of improved properties in a broad range of applications.
\end{abstract}

Keywords: Catalysis, Copper, Decarboxylative, Organocatalysis, Enantioselective

\section{Introduction}

\subsection{Organic Synthesis as Central Science}

With the common perception that organic synthesis is a sufficiently mature field, a question is often arising: why do we continue our research efforts in the development of new synthetic methodologies? This is due to a misperception on the efficiency of many organic reactions or synthetic sequences and consequently on the molecules truly available in a reasonable number of steps.

In a society relying heavily on elaborated scientific technologies, organic molecular objects and processes are everywhere from materials, drugs to energy production or storage (Figure 1). But for improved efficiency, these systems tend to exponentially gain in complexity. Eribulin, an anticancer drug commercialized since 2010 and possessing 19 stereogenic centers is a typical example of highly complex technology possessing optimal activity. However, its industrial preparation requires 62 steps, an effort hard to envisage for most researchers and industrials. ${ }^{[1]}$ While this represents an unprecedented tour de force, in most cases, if a scaffold seems too complicated to prepare, given our actual knowledge, it will likely not be assembled despite its potential properties. Consequently, many scientific fields strongly rely on the continuous improvement in the know-how of organic synthesis and in the improvement of the synthetic toolbox.

Thanks to the global research efforts, we continuously see revolutions in the way we conceive organic transformations such as the advent of $\mathrm{C}-\mathrm{H}$ activation, organocatalysis or more recently the one of photoredox catalysis. Among emerging trends, a major goal over the last years is to move from expensive metal complexes that were widely developed in the 1990 's and try to discover new 3D metal catalyzed transformations.

[a] Dr. A. Quintard

Aix Marseille Univ, CNRS, Centrale Marseille, iSm2, Marseille, France

E-mail: adrien.quintard@univ-amu.fr

Homepage: https://ism2.univ-amu.fr/fr/annuaire/stereo/quintardadrien
In this field, my group recently initiated a research program dedicated to the discovery of new approaches for the rapid synthesis of complex molecules of interest using cheap and widely available iron or copper catalysts. ${ }^{[2]}$ The new reactions can construct rapidly and under mild conditions key building blocks from readily available starting materials. In this account are presented our success at selectively activating ketoacids and notably bio-sourced 1,3-acetonedicarboxylic acid with simple and cheap copper catalysts. Their decarboxylative condensation with aldehydes under exceedingly mild conditions can create more rapidly known natural products scaffolds such as 1,3-polyols. In addition, in order to bypass the inherent selectivity and reactivity issues associated with these catalysts, when necessary, we also often took advantage of multicatalytic combination with organocatalysis. ${ }^{[3]}$ In addition to the rapid preparation of natural product fragments, cascade incorporation of fluorine also provided new type of synthetic analogues of improved properties in a broad range of applications.

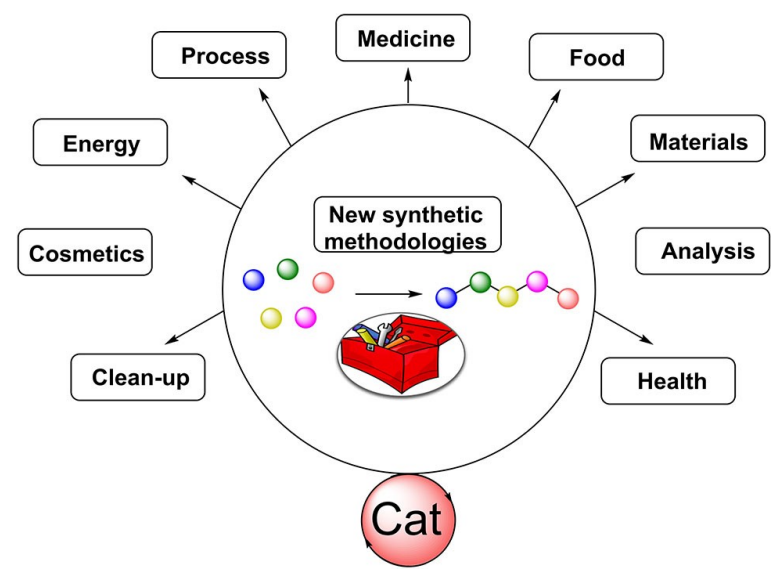

Figure 1. Organic synthesis as central science. 


\subsection{Copper catalyzed Decarboxylative Functionalization of Carbonyl Derivatives}

Carbonyl derivatives are crucial fragments in organic synthesis, often difficult to activate regioselectively and under mild conditions. In order to solve the challenge associated with their functionalization, Nature widely applies decarboxylative transformations. Taking inspiration from Nature, chemists have been able to react in these processes different carbonyl derivatives such as esters, thioesters or ketones, possessing adjacent carboxylic acid functions (Figure 2). The decreased pka at the enolizable position facilitates the deprotonation and the overall functionalization process, driven by the release of $\mathrm{CO}_{2}$ from the product. ${ }^{[4]}$

In order to activate the different reaction components, chemists have relied either on organocatalysis or on metalcatalysis using copper complexes. The breakthrough in this subfield came from the report by the group of Mathew Shair on an efficient thioester decarboxylative aldolization (Scheme 1). ${ }^{[5]}$ Notably, using an appropriate chiral bisoxazolidine ligand/copper salt combination, malonic acid half thioesters could be condensed on aldehydes in excellent diastereo- and enantio-control (Scheme 1.b). In this process, the copper complex generates a reactive enolate under mild conditions and in the absence of exogenous strong bases. Upon condensation with the aldehyde, $\mathrm{CO}_{2}$ exclusion provides the expected aldol adduct.

Following this pioneering work, different copper-catalyzed decarboxylative functionalization were developed. ${ }^{[6]}$ Systems based on achiral or enantiopure ligands could induce the formation of divers ketones or nitriles compounds depicted in Scheme 2. However, despite its great interest in the preparation of useful substrates by using particularly mild conditions, this strategy is clearly underdeveloped and only a limited set of transformations were reported.

Given this potential, we recently got attracted by the design of new strategies, using simple ketoacids for the preparation of key building blocks and natural products fragments. Most notably, use of one of the cheapest ketoacid, 1,3-acetonedicarboxylic acid $\mathbf{1}$, could considerably expand the power of copper catalyzed decarboxylative transformations by

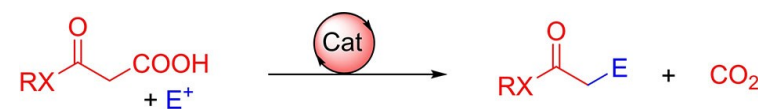

Figure 2. Principle of decarboxylative functionalization.

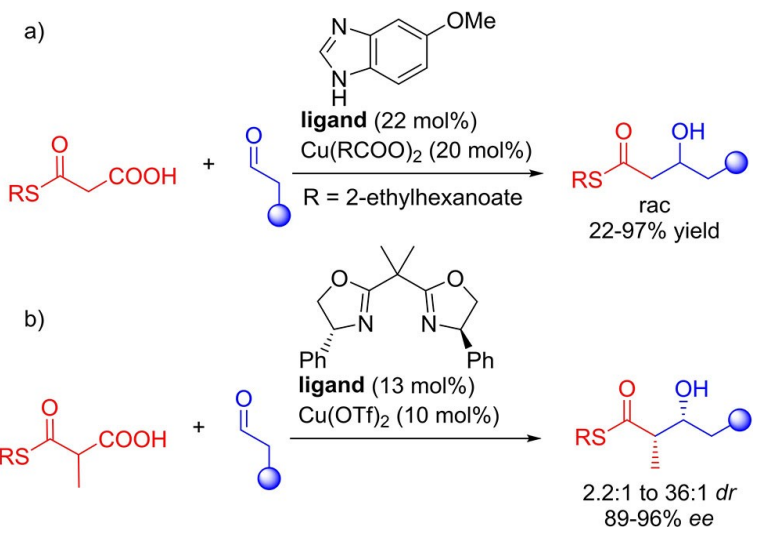

Scheme 1. Discovery of a copper-catalyzed decarboxylative aldolization by the group of Shair.

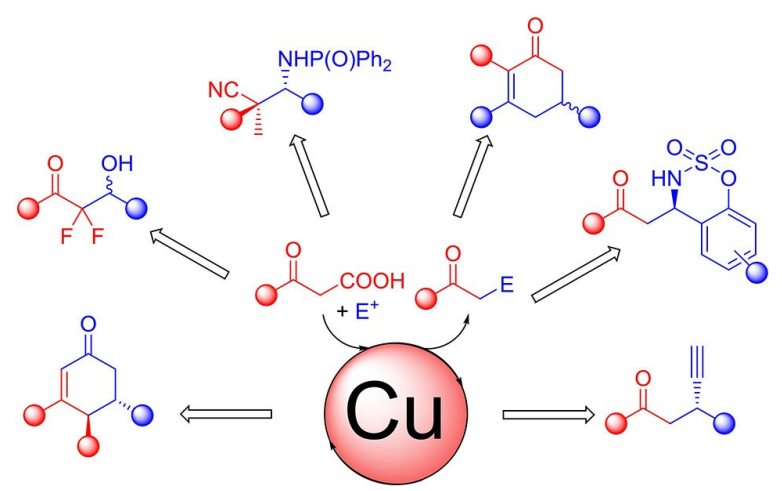

Scheme 2. Literature examples of copper catalyzed decarboxylative functionalization.

inserting a bidirectional dimension to the reactions, increasing

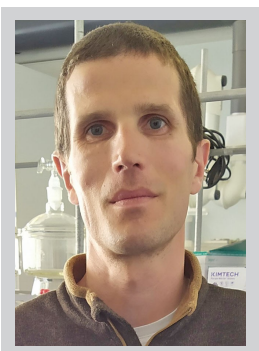

After discovering chemistry at the technical institute of Castres from the University Toulouse III Adrien Quintard obtained a Master degree in 2007 at Lyon (CPE Lyon/University Lyon I). He then moved to Geneva for a PhD obtained in 2011 before a first Postdoc at the university of Stanford. He then joined Marseille in 2012 for a second Postdoc before obtaining in 2013 an ANR starting grant to initiate a new research program on multicatalysis. He subsequently entered the CNRS as a senior researcher in 2014 with research interests encompassing a broad range of areas of organic chemistry (catalysis, synthesis, supramolecular chemistry). Recently, his contribution was recognized by the award of the young researcher Emergence prize from the Organic Division of the French Chemical Society, a Thieme Chemistry Journals award and in 2021 a CNRS bronze medal. 


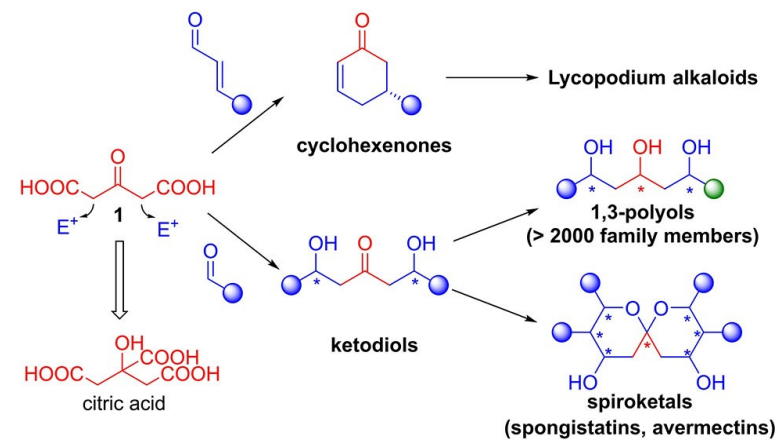

Scheme 3. Overview of the decarboxylative bidirectional strategies.

achievable structural complexity (Scheme 3).

In this context, our recent success at preparing enantioenriched cyclohexenones and ketodiols, key precursors of extended 1,3-polyols will be detailed. As demonstrated along this account, in order to obtain optimized reactivity and selectivity, we also often rely on the application of multicatalysis principles. These new approaches using decarboxylative transformations impressively shortcut the synthesis of these key fragments. Here, we highlight the genesis of this project and its application notably to bioactive molecules synthesis as well as to other fields such as the development of new materials.

\section{Enantioselective Cascade Cyclohexenones Synthesis}

Our journey in this field started with the investigation of the cascade synthesis of enantioenriched cyclohexenones $3{ }^{[7]}$ Such molecules had been applied in the total synthesis of divers natural products such as Lyconadin A, Lannotinidine B or Fawcettimine, but required multiple-steps for their synthesis. ${ }^{\left[{ }^{[8]}\right.}$ In order to shortcut their preparation, the idea was to apply an intriguing bio sourced reagent, 1,3-acetonedicarboxylic acid $\mathbf{1}$, as an alternative acetone surrogate potentially able to directly generate the desired cyclohexenones. This diacid, applied in the famous Robinson Tropinone synthesis is prepared in kilogram scale from citric acid ${ }^{[9]}$ and can react in a bidirectional manner upon double decarboxylation. However, its higher reactivity is also linked to a lower stability often providing acetone as side product through competing decarboxylative protonation. As a result, selectively activating it in enantioselective transformations represented an unsolved challenge.

In our planned strategy, the ketodiacid $\mathbf{1}$ should react selectively through a Michael addition on an $\alpha, \beta$-unsaturated aldehyde $\mathbf{2}$ followed by intramolecular crotonization
(Scheme 4). In order to promote the enantioselective Michael addition, we first assumed that a chiral pyrrolidine aminocatalyst cat1 should be sufficient to activate the aldehyde and promote an efficient direct ketoacid addition. However, eventhought a decent enantiocontrol was observed $(90 \% \mathrm{ee})$, the reactivity remained modest and was associated with large amount of acetone formation ( $12 \%$ yield).

In order to facilitate the nucleophilic addition, we then hypothesized that addition of a co-catalyst could enable a dual activation where both nucleophile and electrophile would be appropriately organized. Indeed, in recent years, considerable progress was obtained through the appropriate merging of complementary metal- and organo-catalysis. ${ }^{[3]}$

Among all the co-catalysts tested, we discovered that addition of simple iron or copper Lewis acids could considerably improve both reactivity and selectivity. Notably, using $6 \mathrm{~mol} \%$ of $\mathrm{Cu}(i-\mathrm{BuCOO})_{2}, 3 \mathbf{a}$ could be isolated in $50 \%$ yield and $>98 \% e e$, indicating that the copper complex was involved in the rate and enantio-determining step of the process. Use of the ketodiacid is crucial for both 1,4 addition and crotonization since when using a mono ketoacid, the reaction stopped at the Michael addition while no cyclization was observed.

Through this multicatalyzed protocol, substituted cyclohexenones possessing different aromatic rings could be prepared directly from commercial products and with excellent enantiocontrol ( $96 \% e e$, Scheme 5). For example, the reaction tolerated electron-donating or electron-withdrawing substituents at different positions of the aromatic. Replacing the aromatic by an ester was also possible generating $3 \mathbf{e}$ in $94 \% \mathrm{ee}$ while aliphatic chains were less tolerated providing only minor amount of the corresponding cyclohexenones.

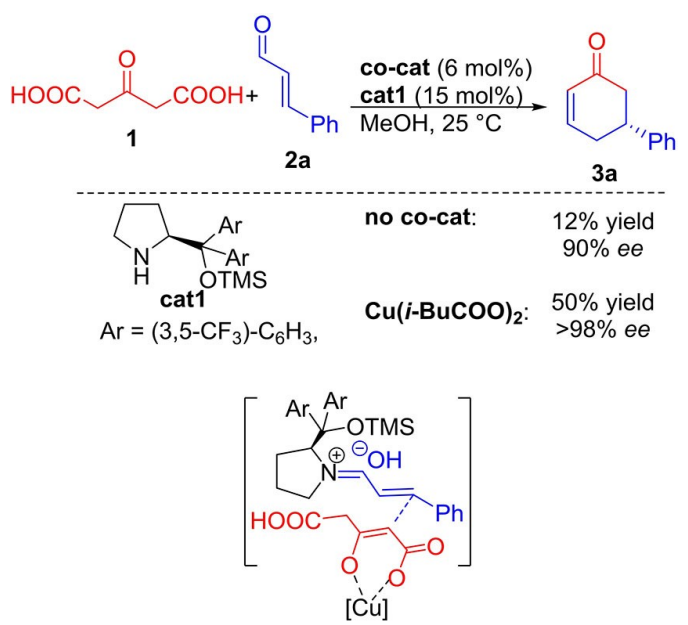

Scheme 4. Optimization of the cascade cyclohexanone synthesis. 


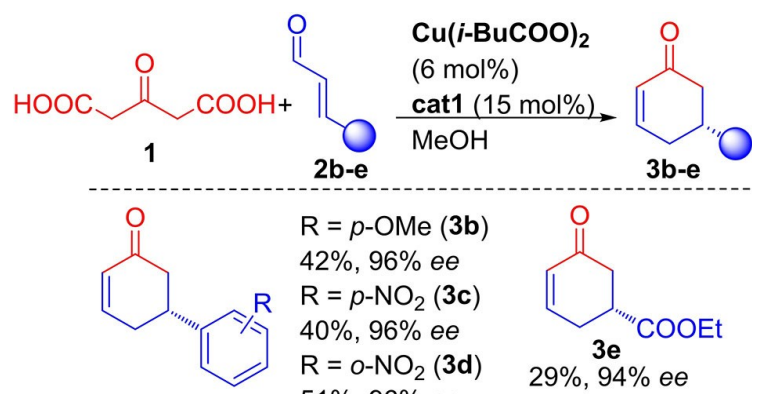

$51 \%, 96 \%$ ee

Scheme 5. Example of cyclohexenones synthesized from 1

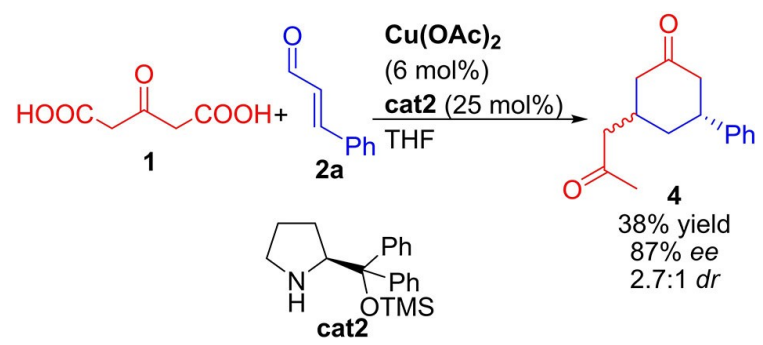

Scheme 6. Cascade addition of $\mathbf{1}$ to $\alpha, \beta$-unsaturated aldehyde $\mathbf{2}$ a.

Interestingly, modifying conditions notably by using an excess of $\mathbf{1}$ in the presence of cat 2 and $6 \mathrm{~mol} \%$ of $\mathrm{Cu}(\mathrm{OAc})_{2}$, a cascade Michael addition on the in-situ generated enone was observed (Scheme 6). Through this cascade process, 3 new $\mathrm{C}-\mathrm{C}$ bonds could be generated in an overall $38 \%$ yield and $87 \%$ ee, demonstrating the potential of this approach for complex molecules synthesis.

\section{1,3 Polyols Synthesis}

\subsection{Interest and Strategy}

With this first bidirectional transformation in hand, we then wondered about applying $\mathbf{1}$ in the synthesis of crucial 1,3polyols. Indeed, this 1,3-polyol motif is present in a variety of natural products and drugs but its preparation is most of the time associated with lengthy stepwise synthesis. ${ }^{[10]}$ Given the bioactivity profile of molecules possessing this scaffold, it would be highly desirable to discover a straightforward access from readily available substrates. More-over, a strategy also amenable to structural modification potentially tuning molecules properties would be of additional interest.

In this context, condensing two molecules of aldehydes with $\mathbf{1}$, should readily generate the desired key ketodiols in one single cascade (Scheme 3). The ketodiol motif is present in different natural products but most interestingly, ketodiols are also the direct precursors of extended 1,3-polyols upon ketones reduction. As a result, discovering an efficient bidirectional condensation of $\mathbf{1}$ to two molecules of aldehydes should be of great benefit. More-over, based on this cascade, it should also be possible to easily modulate the aldehydes structures to easily tune polyols side chains.

\subsection{First Results on Bidirectional Aldolization}

Given Shair's development on copper catalyzed aldolization ${ }^{[5]}$ and our own success at using achiral copper salts to activate $\mathbf{1}$, we attempted to develop a direct enantioselective bidirectional aldolization. However, condensing 1 with propionaldehyde using $\mathrm{Cu}(\mathrm{OTf})_{2}$ and $\mathbf{L 1}$, ketodiol $\mathbf{6}$ was isolated in poor yield and as a racemate (Scheme 7.a). Optimizing the conditions notably by applying $\mathrm{Cu}(i-\mathrm{BuCOO})_{2}, 6$ could be generated albeit in moderate $32 \%$ yield, $1.1: 1 d r$ and $40 \%$ ee (Scheme 7.b). These modest results were rather intriguing and could be explained by a strong coordination of the ketodiacid 1, ejecting the chiral ligand from the coordination sphere of the metal (Scheme 7.c). As a result of this strong coordination, confirmed by UV monitored titration, a new achiral copper complex can be generated in-situ and catalyze the reaction considerably decreasing the enantioselectivity of the transformation.

However, this result also indicated that potentially, use of simple Lewis acids could promote rapidly the bidirectional process generating racemic ketodiols under mild conditions. This was indeed the case, and applying $15 \mathrm{~mol} \%$ of cheap $\mathrm{Cu}(\mathrm{acac})_{2}$ or $\mathrm{Fe}(\mathrm{acac})_{3}$ could trigger the formation of a broad range of ketodiols (Scheme 8). ${ }^{[1]}$

The reaction occurs at room temperature and in the absence of base to generate diversely substituted ketodiols of interest. For example, $\mathbf{6} \mathbf{d}$, a direct precursor of natural product ericanone, ${ }^{[12]}$ could be prepared in one single step with $39 \%$

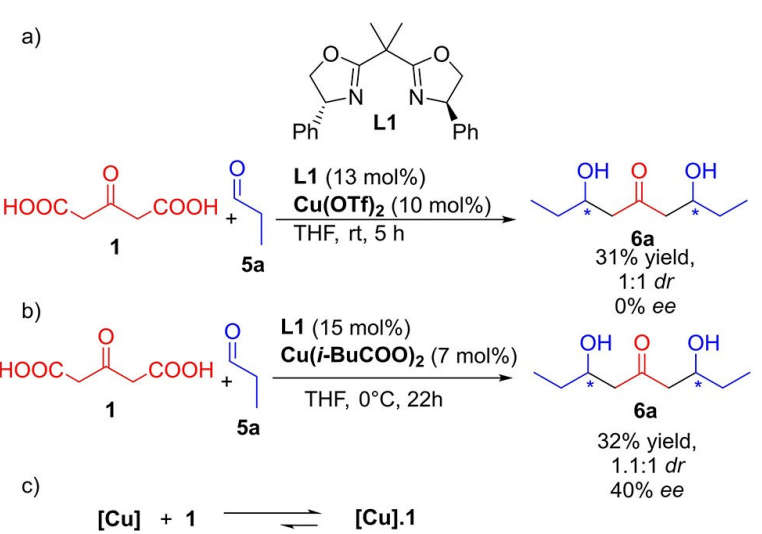

Scheme 7. Preliminary attempts at enantioselective bidirectional aldolization. 


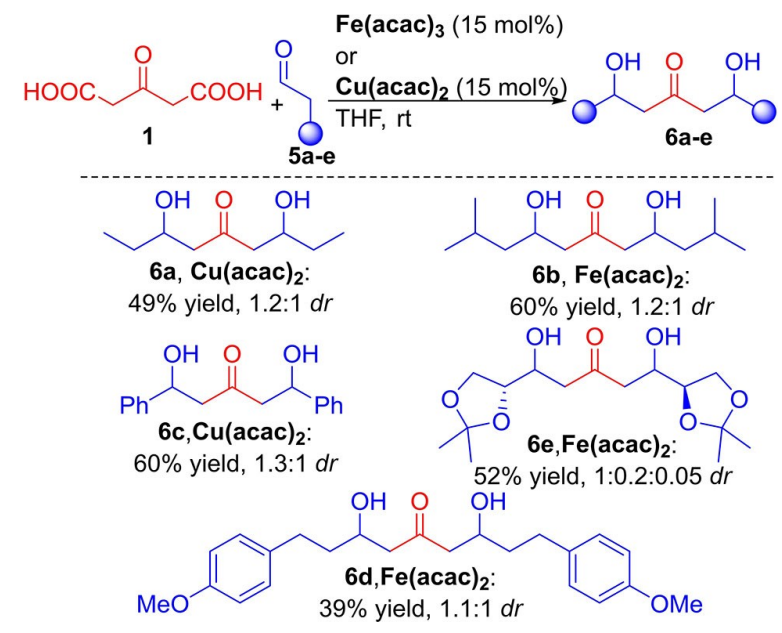

Scheme 8. Bidirectional aldolization using simple Lewis acids.

yield. In all cases, the diastereoselectivity remained modest, indicating that the first generated stereocenter did not control the second aldolization event. However, when starting from an appropriate chiral aldehyde, a diastereoselective process controlling the generation of the two new stereocenters was observed. Consequently, the major diastereomer of $\mathbf{6 e}$ could be generated in $75 \%$.

The copper-catalyzed bidirectional aldolization follows the general mechanism depicted in Scheme 9. This reaction differs

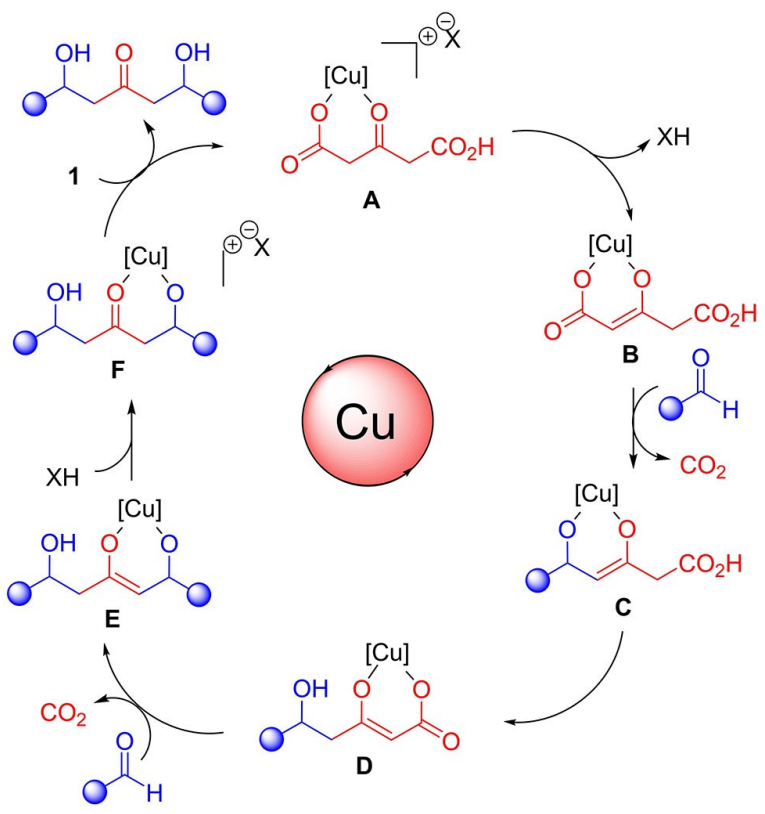

Scheme 9. Mechanism of the bidirectional decarboxylative aldolization. from previous copper based process by the absence of base. Upon addition of the ketodiacid, enolate $\mathbf{B}$ is generated from A under mild conditions. This enolate reacts with the first equivalent of aldehyde forming $\mathbf{C}$ after decarboxylation $\left(-\mathrm{CO}_{2}\right)$. Following the same sequence of aldolization/decarboxylation, the copper alcoholate $\mathbf{F}$ is created. Decoordination of the substrate upon insertion of a new molecule of ketodiacid closes the catalytic cycle.

From this study, we had shown that simple and cheap metal complexes could efficiently activate $\mathbf{1}$ towards bidirectional aldolization. It also appeared that two different approaches could be envisaged to trigger the formation of enantioenriched ketodiols: 1) use of more electrophilic aldehydes facilitating the enantioselective process prior to chiral ligand decoordination. 2) use of enantioenriched aldehydes in a diastereoselective aldolization.

\subsection{Enantioselective Bidirectional Aldolization on Perfluorinated Aldehydes}

As mentioned earlier, there is a clear interest at modifying 1,3polyols scaffolds in order to modulate their properties. Given that their bioactivity is in great part due to the formation of strong hydrogen bonding frameworks, improving its strengths represents a promising strategy. ${ }^{[13]}$ In this context, we were attracted by the preparation of extended polyols, possessing perfluorinated side chains. ${ }^{[14]}$ Indeed, incorporation of these electron-withdrawing chains, would provide strong chiral hydrogen bonding frameworks, attractive notably in the context of chiral anion recognition.

According to the results shown on bidirectional aldolization, condensation of $\mathbf{1}$ on highly electrophilic perfluorinated aldehydes could be fast enough to avoid chiral ligand decoordination prior to the crucial enantioselective event.

Confirming our hypothesis, different highly electrophilic halogenated aldehydes reacted with excellent enantiocontrol using bis-oxazolidine ligand L1 (Scheme 10). ${ }^{[15]}$ This bidirectional aldolization using $\mathbf{1}$, provided perfluorinated ketodiols 10 a-c in $11.5: 1 d r$ to $32: 1 d r$ and $>96 \%$ ee. Using chloral $\mathbf{7 d}$, chlorinated ketodiol 11 was also prepared with good efficiency $(4: 1 d r$ and $90 \% e e)$. The reaction was also efficient starting from simple ketoacids and among the products, aldol adducts 12 and 13 were also generated in more than $80 \% \mathrm{ee}$.

As mentioned earlier, the ketodiols can easily be reduced using $\mathrm{NaBH}_{4}$, affording the corresponding chiral 1,3,5-triols such as $\mathbf{1 4 c}$ (Scheme 11.a). Single crystal X-ray diffraction of $14 \mathrm{c}$ confirmed the absolute configuration of the obtained products. Limited steric repulsion between the halogenated chain and the phenyl substituent of the ligand during the C-C bond formation, account for the excellent selectivity observed in the transition state (Scheme 11.b). 


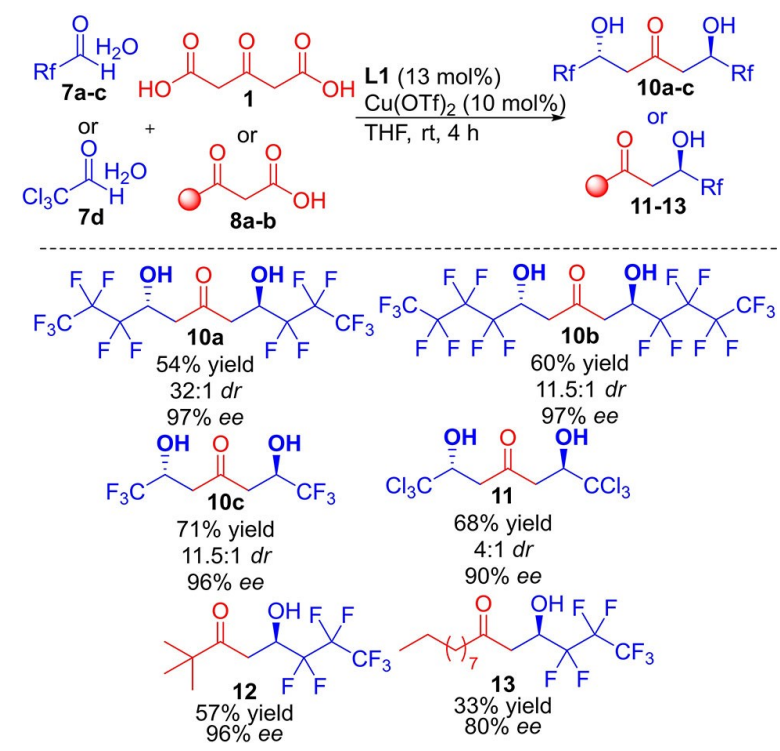

Scheme 10. Decarboxylative aldolization on electrophilic halogenated aldehydes.

a) Triol structure:

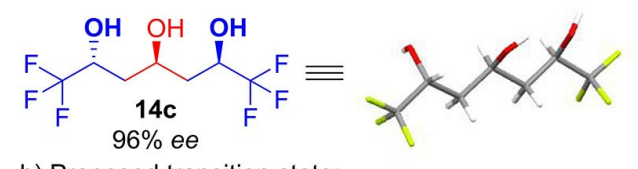

b) Proposed transition state:

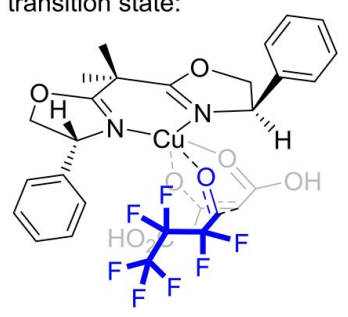

Scheme 11. Single crystal X-Ray analysis of $\mathbf{1 4} \mathrm{c}$ and proposed transition state.

\subsection{Enantioselective Fluorination/Aldolization}

Aside from the strategy of direct enantioselective aldolization on highly electrophilic aldehydes, the second complementary strategy consisted in performing a diastereoselective aldolization on enantioenriched aldehydes. Fluorinated aldehydes are peculiar substrates easily generated in excellent enantiocontrol using aminocatalysis but suffering from high instability (Scheme 12). As a result, literature protocols involve their derivatization to the corresponding alcohols upon treatment with $\mathrm{NaBH}_{4} \cdot{ }^{[16]}$ From a synthetic perspective, it would be highly desirable to intercept these species through diastereose-

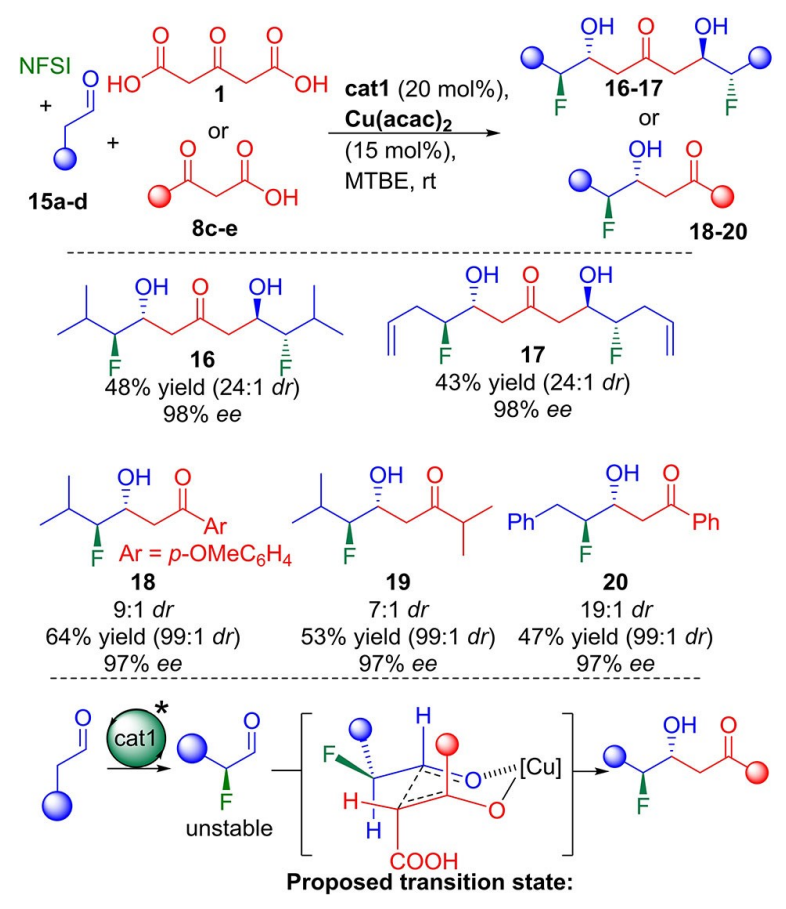

Scheme 12. Representative scope of the multicatalyzed fluorination-aldolization.

lective aldolization. Indeed, after ketone reduction, fluorinated polyols of potential enhanced properties would be readily generated. While selective incorporation of fluorine into extended polyols looked promising notably to improve their bioactivity, ${ }^{[17]}$ only few methods based on stepwise processes were available to access a limited set of fluorinated scaffolds. ${ }^{[18]}$

Several challenges were to be solved in order to develop an efficient fluorination/aldolization protocol. The $\mathrm{C}-\mathrm{C}$ bond formation should be compatible with the halogenation conditions, fast and occurring under mild conditions to avoid intermediate aldehyde decomposition/racemization.

Solving all these issues, we discovered that an appropriate multicatalytic strategy could generate the expected mono- or bidirectional cascade products in excellent stereocontrol (typically $>9: 1 d r$ and $97 \% e e$; Scheme 12 ). ${ }^{[19]}$ The sequence is based on an enantioselective organocatalyzed fluorination of aldehydes 15 with NFSI as the electrophilic source of fluorine using aminocatalyst cat1. Upon chiral aldehyde generation, the copper catalyst activates the ketodiacid promoting the diastereoselective aldolization. Dipole-dipole interactions minimization enable to obtain excellent diastereocontrol during the transition state. The scope of this transformation is particularly broad, with all kind of ketone donors or aldehyde acceptors tolerated (Scheme 12). Most notably, using ketodiacid 1, ketodiols featuring four controlled acyclic stereogenic 
centers, a substantial synthetic challenge, are formed with excellent levels of stereocontrol. In all cases, the appropriate combination between $\mathrm{Cu}(\mathrm{acac})_{2}$ and cat1 is crucial both for reactivity and selectivity, demonstrating the power of multicatalysis.

\subsection{Synthetic Application of the Halogenation/Aldolization}

The bidirectional approach is particularly interesting to prepare rapidly complex scaffolds of interest. Notably, from 17, generated in one single step from commercial reagents, reduction of the central ketone and subsequent desymetrization upon formation of the thermodynamic syn-acetal, afforded 21 possessing differentiated terminal alkenes (Scheme 13).

As a result, upon treatment with iodine, selective iodoetherification generates 22, possessing 7 stereogenic centers and in only 4 steps from commercial substrates.

With an efficient method in hand for the preparation of fluorinated polyols, we demonstrated its relevance in a concise synthesis of the C15-C27 fragment of bastimolide A (Scheme 14). ${ }^{[20]}$

Bastimolide $\mathrm{A}$ is a naturally occurring polyol possessing potent antimalarial activity. ${ }^{[21]}$ Its structure notably features a challenging pseudo-symmetric C15-C27 polyol fragment. Performing the bidirectional fluorination/aldolization using protected aldehyde $\mathbf{2 3}$, upon ketone reduction, the fluorinated polyol fragment 24 could be isolated with excellent stereocontrol (19:1 dr, >95\% ee). In order to compare bioactivities, we also applied a chlorination/aldolization strategy to the preparation of a chlorinated analogue. ${ }^{[22]}$ Following the Lprolinamide catalyzed enantioselective chlorination of the aldehyde, ${ }^{[23]}$ bidirectional aldolization and ketone reduction provided chlorinated polyol 25 . From 25, radical promoted chlorine removal following Britton's group approach ${ }^{[22]}$ easily provided the natural polyol chain 26. Removal of the silyl protecting group under acidic conditions gave access to all natural, fluorinated and chlorinated $\mathrm{C} 15-\mathrm{C} 27$ fragments of

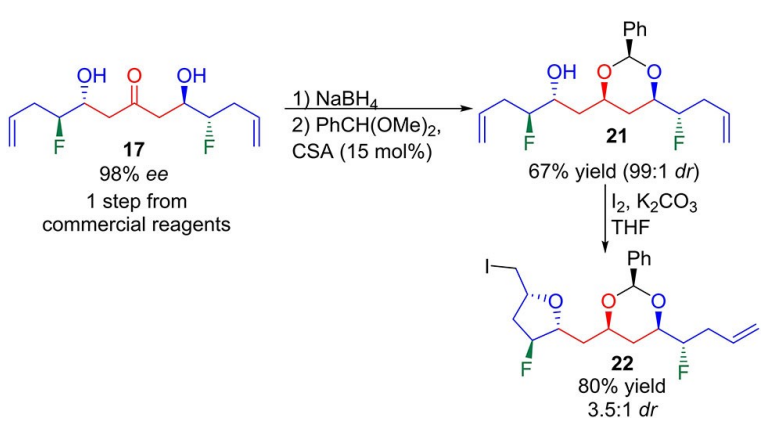

Scheme 13. Desymetrization of 17

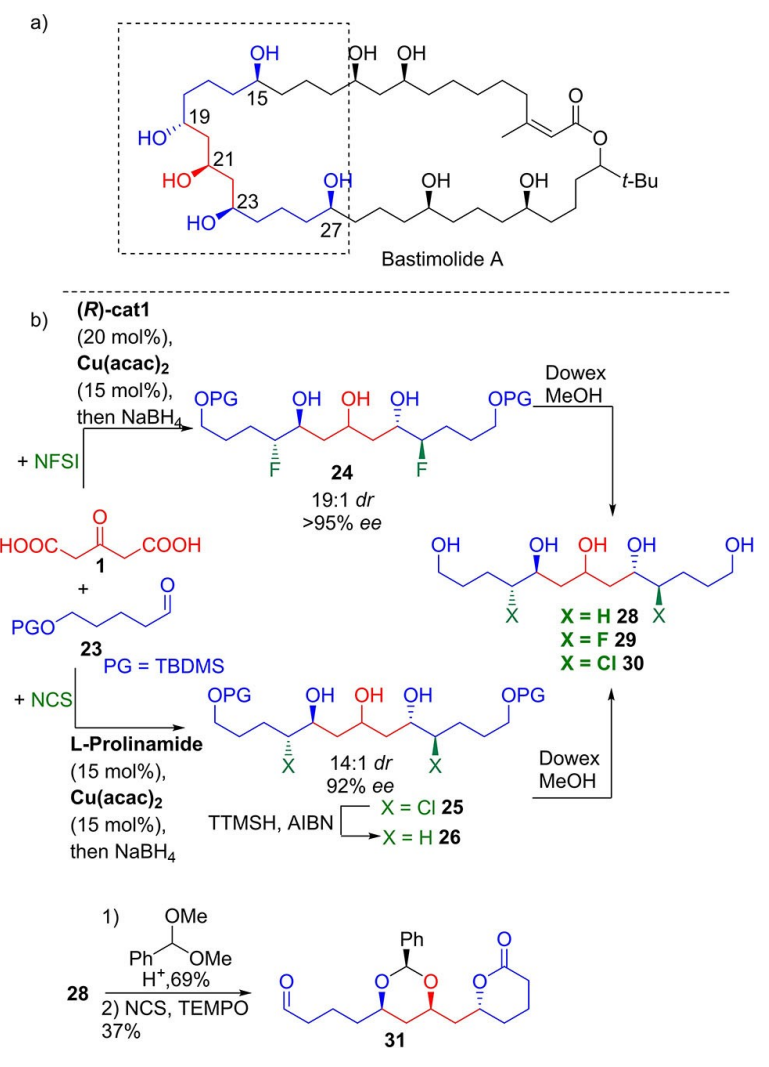

Scheme 14. Enantioselective synthesis of $\mathrm{H}, \mathrm{F}, \mathrm{Cl}$ analogue fragments of bastimolide A.

bastimolide A. Once again, in order to breake molecular symmetry and obtain an advanced synthetic intermediate, the thermodynamically more stable syn-acetal was generated and subjected to oxidation using NCS/TEMPO. As a result of this desymetrization, one alcohol was oxidized to the aldehyde and the other to the cyclic lactone, generating 31, an advanced synthetic intermediate of bastimolide A.

\subsection{Aldehydes Bis-fluorination/Decarboxylative Aldolization}

Aside from bidirectional systems, our above studies demonstrated the excellent behavior of simple copper salts to activate ketoacids under particularly mild conditions compatible with multicatalyzed processes. These catalytic properties pushed us to study application in the development of other multicatalyzed transformations.

For example, the aldehyde fluorination/decarboxylative aldolization could also be extended to the preparation of gemdifluorinated $\beta$-aldol building blocks (Scheme 15). ${ }^{[24]}$ Modification of the conditions by using 2 equivalents of NFSI and a 


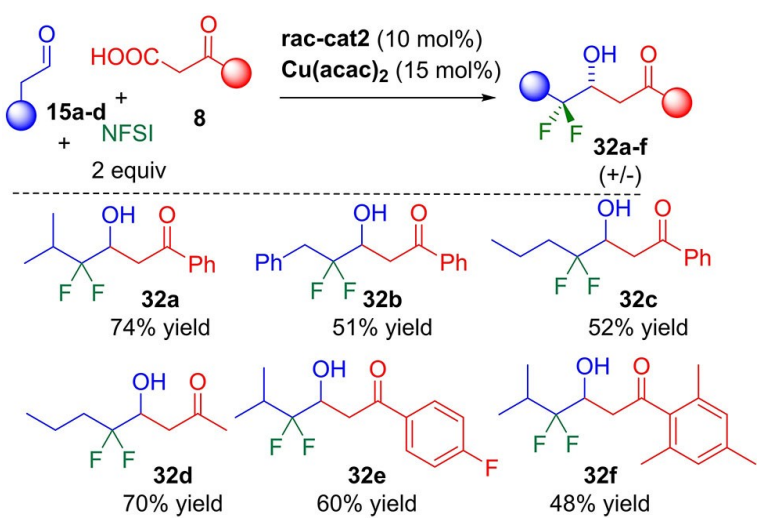

Scheme 15. Representative scope for the bis-fluorination/decarboxylative aldolization.

racemic aminocatalyst (rac-cat2) generated the desired gemdifluorinated aldehyde. ${ }^{[25]}$ In-situ intercepting this aldehyde using a ketoacid and $\mathrm{Cu}(\mathrm{acac})_{2}$, the desired bis-fluorinated adducts $32 \mathbf{a}-\mathbf{f}$ could be prepared in $31-74 \%$ yield. Given the achiral nature of the aldehyde intermediate, in contrast to the mono-fluorination/aldolization sequence, here, the adducts are obtained as racemates. All attempts at performing an enantioselective aldolization using chiral catalysts failed. However, kinetic resolution of the corresponding diols could provide the desired enantioenriched products. ${ }^{[26]}$ These molecules represent original scaffolds and are easily prepared from simple building blocks and under mild conditions. Given the presence of the gem-difluoro fragment in numerous drugs, insertion of such scaffolds might also be interesting to modulate bioactive molecules properties.

\subsection{Oxa-Michael/decarboxylative aldolization sequence}

Aside from halogenated scaffolds, multicatalysis could also be attractive to intercept other classes of sensitive aldehydes prior to their decomposition. Upon aminocatalyzed addition of oximes to $\alpha, \beta$-unsaturated aldehydes 34 , unstable enantioenriched aldehydes are generated (Scheme 16). ${ }^{[27]}$

Once again, we hypothesized that, upon suitable multicatalytic activation, decarboxylative aldolization using ketoacids could unlock the access to other highly useful nonsymmetric polyols (Scheme 16). ${ }^{[28]}$

Among the numerous catalysts tested for the aldolization step, $\mathrm{Cu}(i-\mathrm{BuCOO})_{2}$ provided the best efficiency and the adducts of the multicomponent assembly could be isolated in $48-67 \%$ yield.

As a limitation, while fluorinated aldehydes provided excellent diastereocontrol, here, the remote stereogenic center only provided modest chiral induction. As a result, while the enantioselectivities arising from the use of cat1 were above

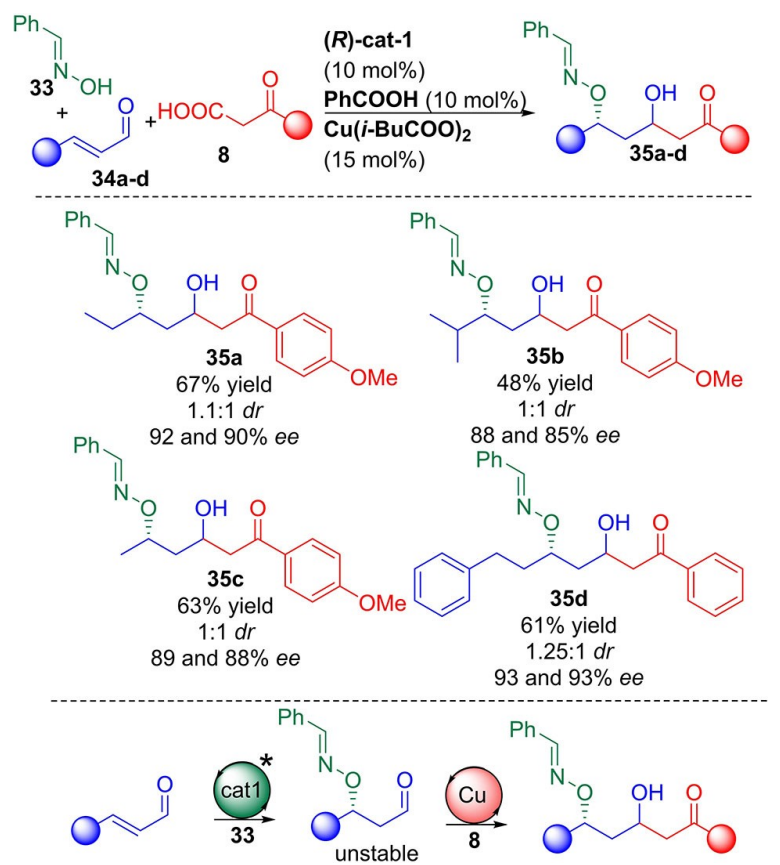

Scheme 16. Representative scope for the oxa-Michael/decarboxylative aldolization.

$85 \% e e$, the multicatalysis adducts could only be isolated in modest diastereocontrol (around 1.1:1 dr). However, despite this aspect, the sequence is of great interest to access such structures rapidly and its power was demonstrated in the concise synthesis of Yashabushitriol, a naturally occurring triol (Scheme 17). ${ }^{[29]}$

From 35d, obtained in $93 \%$ ee, dibal-H promoted diastereoselective reduction and diastereomers separation provided 36. Final oxime hydrogenation gave the expected yashabushitriol as $95 \%$ of the major diastereomer. Overall,

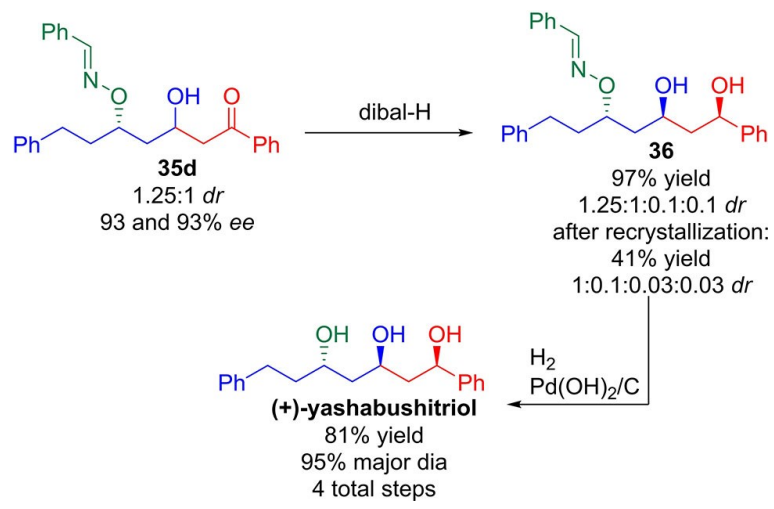

Scheme 17. Yashabushitriol synthesis. 
yashabushitriol is prepared in only four steps, a much shorter route than the literature previous ones requiring 9 to 11 steps. ${ }^{[30]}$ This considerable step economy, demonstrates the power of multicatalysis and of the copper catalysts activation of ketoacids to considerably shorten natural products synthesis.

\section{Supramolecular Properties of the Halogenated Polyols Structures}

As mentioned in the introduction, developing new synthetic tools can enable to access otherwise inconceivable molecular structures possessing innovative properties. Thanks to the cascades developed, new types of fluorinated and chlorinated polyols could be easily prepared. In order to study the impact of this halogenation, we notably studied their chloride anion binding properties (Scheme 18.a). ${ }^{[31]}$ While moderate binding was observed for the naked triol 36a $\left(133 \mathrm{M}^{-1}, \mathrm{X}=\mathrm{H}\right)$, insertion of fluorine or chloride considerably improved this coordination (413 and $617 \mathrm{M}^{-1}$ ). This indicates a stronger triol hydrogen bonding framework which might be of great importance for the design of bioactive molecules incorporating this fragment.

Organogels are another class of supramolecular assembly of interest. Upon formation of self-assembled fibers, organogelators are able to entrap solvents in a strong $3 \mathrm{D}$ matrix creating new type of materials. ${ }^{[32]}$ Triols $\mathbf{3 6} \mathbf{a}-\mathbf{c}$, easily prepared by the bidirectional aldolization could be attractive to prepare new type of organogels (Scheme 18-b). Their central strong Hbonding framework and external lipophilic chains could stabilize the self-assembly entrapping organic solvents. In the absence of halogen atoms, no gelation was observed while weak gelation was induced with $36 \mathrm{c}$ possessing chlorine substituents. In sharp contrast, fluorinated $\mathbf{3 6} \mathbf{b}$ generated particularly strong gel for such low-molecular weight molecules. ${ }^{[31]}$ This impressive behavior results from the beneficial role of the fluorine atoms over several parameters. It strengthens the central hydrogen-bonding framework, introduces a conformational bias as well as supplementary lipophilic interactions, rigidifying the material.

Concerning the perfluorinated 1,3,5-triols synthesized, they also provided excellent anion coordination and for example, 14 coordinated to $\mathrm{Cl}^{-}$with $\mathrm{K}=4400 \mathrm{M}^{-1}$. ${ }^{[15]}$ In addition, these perfluorinated triols were also able to recognize enantioselectively chiral anions with a selectivity factor of 4.5. These excellent anion binding abilities and selectivities are due to the presence of the lateral fluorinated chains, able to increase the acidity of the triol core. These properties were also applied in $\mathrm{H}$-bond donor catalysis, notably in the opening of epoxides with $\mathrm{CO}_{2} \cdot{ }^{[33]}$ Using only $1 \mathrm{~atm}$ of $\mathrm{CO}_{2}$, valuable carbonates could be generated using only $5 \mathrm{~mol} \%$ of triol as organocatalyst.
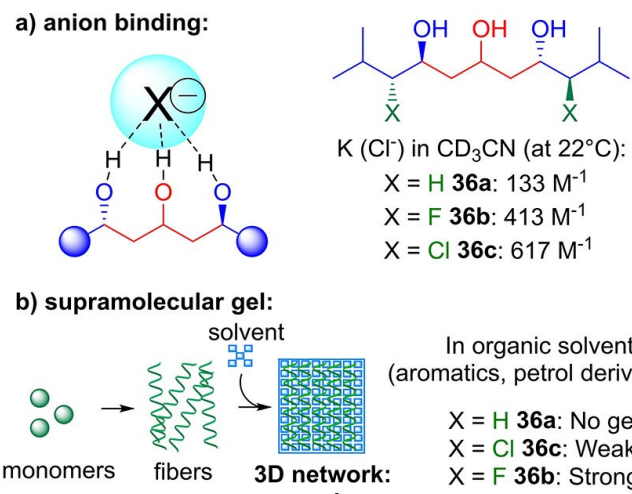

In organic solvents (aromatics, petrol derivatives)

$X=H$ 36a: No gel $X=\mathrm{Cl}$ 36c: Weak gel gel

c) perfluorinated triols:

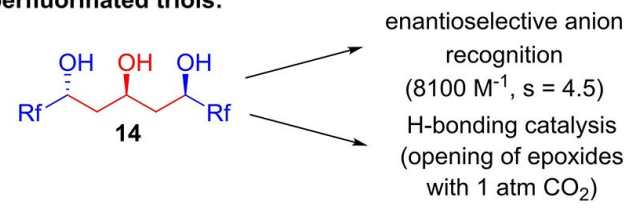

Scheme 18. Supramolecular properties of halogenated triols.

All these examples, demonstrate that thanks to the design of new organic transformations, it is possible to create innovative scaffolds, previously inaccessible, efficient in a wide range of applications.

\section{Conclusion}

We are in a world based on organic synthesis for almost all fields ranging from health, energy to materials. In order to feed the world with innovations, we are continuously relying on the design of more complex organic scaffolds which only more efficient and sustainable organic transformations can build. Consequently, we should not stop but intensify our efforts at developing new synthetic methodologies.

In this context, use of ketoacids and notably bio-sourced 1,3-acetonedicarboxylic acid, appropriately activated by cheap copper catalysts, represents an attractive field of research. Condensation with aldehydes under exceedingly mild conditions can create more rapidly known natural products scaffolds such as 1,3 polyols. More-over selective methods for fluorine insertion also provided new types of synthetic analogues of improved properties in a broad range of applications (anion binding, materials, catalysis). In addition, when limited by the copper catalyst activity, applying multicatalysis considerably improved reaction efficiency. As a result, multicatalysis enabled to design new transformations easily combining multiple components directly into complex scaffolds of interest. 
Given its potential, the future should see more strategies based on copper catalyzed mild activation of ketoacids. Most notably, it should be the approach of choice to functionalize ketones by replacing traditional stoichiometric reagents based on expensive or pyrophoric organometallic bases. It should also be a valuable tool to enhance selectivity in known functionalization of ketones as for example in challenging aldolization or Mannich type transformations.

\section{Acknowledgements}

All co-workers involved in this adventure are warmly acknowledged, notably Prof. Jean Rodriguez, Dr. Céline Sperandio and Miss Angela Riccuci. The Centre National de la Recherche Scientifique (CNRS) and Aix-Marseille Université are warmly acknowledged for financial support.

\section{References}

[1] a) H. Ledford, Nature 2010, 468, 608; b) K. C. Nicolaou, Tetrahedron 2003, 59, 6683.

[2] See for example: a) A. Quintard, T. Constantieux, J. Rodriguez, Angew. Chem. Int. Ed. 2013, 52, 12883; b) M. Roudier, T. Constantieux, A. Quintard, J. Rodriguez, ACS Catal. 2016, 6, 5236.

[3] For general reviews on multicatalysis, see: a) A. E. Allen, D. W. C. MacMillan, Chem. Sci. 2012, 3, 633; b) C. Zhong, X. Shi, Eur. J. Org. Chem. 2010, 2999; c) R. C. Wende, P. R. Schreiner, Green Chem. 2012, 14, 1821; d) N. T. Patil, V. S. Shinde, B. Gajula, Org. Biomol. Chem. 2012, 10, 211; e) Z. Du, Z. Shao, Chem. Soc. Rev. 2013, 42, 1337; f) S. M. Inamdar, V. S. Shinde, N. T. Patil, Org. Biomol. Chem. 2015, 13, 8116; g) S. Afewerki, A. Córdova, Chem. Rev. 2016, 116, 13512; h) F. Romiti, J. del Pozo, P. H. S. Paioti, S. A. Gonsales, X. Li, F. W. W. Hartrampf, A. H. Hoveyda, J. Am. Chem. Soc. 2019, 141, 17952; i) S. P. Sancheti, Urvashi, M. P. Shah, N. T. Patil, ACS Catal. 2020, 10, 3462; j) A. Quintard, Chem. Eur. J. 2021, 27, 89; k) A. Quintard, Isr. J. Chem. 2021, DOI: $10.1002 /$ ijch.202000018.

[4] For general reviews, see: a) Y. Pan, C.-H. Tan, Synthesis 2011, 13, 2044; b) Z. L. Wang, Adv. Synth. Catal. 2013, 355, 2745; c) S. Nakamura, Org. Biomol. Chem. 2014, 12, 394.

[5] a) G. Lalic, A. D. Aloise, M. D. Shair, J. Am. Chem. Soc. 2003, 125, 2852; b) D. Magdziak, G. Lalic, H. M. Lee, K. C. Fortner, A. D. Aloise, M. D. Shair, J. Am. Chem. Soc. 2005, 127, 7284; c) K. C. Fortner, M. D. Shair, J. Am. Chem. Soc. 2007, 129, 1032; In parallel, the group of Cozzi also developed a decarboxylative aldolization albeit with lower efficiency: d) S. Orlandi, M. Benaglia, F. Cozzi, Tetrahedron Lett. 2004, 45, 1747.

[6] a) L. Yin, M. Kanai, M. Shibasaki, J. Am. Chem. Soc. 2009, 131, 9610; b) L. Yin, M. Kanai, M. Shibasaki, Tetrahedron.
2012, 68, 3497; c) F.-L. Zhu, Y. Zou, D.-Y. Zhang, Y.-H. Wang, X.-H. Hu, S. Chen, J. Xu, X.-P. Hu, Angew. Chem. Int. Ed. 2014, 53, 1410; d) H.-Y. Xiong, Z.-Y. Yang, Z. Chen, J.-L. Zeng, J. Nie, J.-A. Ma, Chem. Eur. J. 2014, 20, 8325 ; e) H.-X. Zhang, J. Nie, H. Cai, J.-A. Ma, Org. Lett. 2014, 16, 2542; f) C.-M. Jia, H.-X. Zhang, J. Nie, J.-A. Ma, J. Org. Chem. 2016, 81, 8561 ; g) H.-Y. Xiong, Z.-Y. Yang, Z. Chen, J.-L. Zeng, J. Nie, J.-A. Ma, Chem. Eur. J. 2014, 20, 8325; h) J.-W. Yuan, S.-N. Liu, W.-P. Mai, Org. Biomol. Chem. 2017, 15, 7654; i) J. Lee, S. Wang, M. Callahan, P. Nagorny, Org. Lett. 2018, 20, 2067.

[7] A. Quintard, J. Rodriguez, Chem. Commun. 2015, 51, 9523.

[8] a) H. M. Ge, L.-D. Zhang, R. X. Tan, Z.-J. Yao, J. Am. Chem. Soc. 2012, 134, 12323; b) X.-M. Zhang, H. Shao, Y.-Q. Tu, F.-M. Zhang, S.-H. Wang, J. Org. Chem. 2012, 77, 8174; c) K. Xu, B. Cheng, Y. Li, T. Xu, C. Yu, J. Zhang, Z. Ma, H. Zhai, Org. Lett. 2014, 16, 196; For a two step process, see: d) A. Carlone, M. Marigo, C. North, A. Landa, K. A. Jørgensen, Chem. Commun. 2006, 4928.

[9] R. Robinson, J. Chem. Soc. Trans. 1917, 111, 762.

[10] For reviews, see: a) J. Staunton, K. J. Weissman, Nat. Prod. Rep. 2001, 18, 380; b) S. E. Bode, M. Wolberg, M. Müller, Synthesis 2006, 4, 557; c) B. Schetter, R. Mahrwald, Angew. Chem. Int. Ed. 2006, 45, 7506; d) D. Herkommer, B. Schmalzbauer, D. Menche, Nat. Prod. Rep. 2014, 31, 456; e) P. Kumar, D. Tripathi, B. M. Sharma, N. Dwivedi, Org. Biomol. Chem. 2017, 15, 733; f) C. Sperandio, J. Rodriguez, A. Quintard, Org. Biomol. Chem. 2020, 18, 1025.

[11] A. Quintard, J. Rodriguez, Chem. Eur. J. 2015, 21, 14717.

[12] L. C. Dias, P. K. Kuroishi, E. C. Polo, E. C. de Lucca Jr., Tetrahedron Lett. 2013, 54, 980.

[13] For a recent review, see: a) S. A. Boer, E. M. Foyle, C. M. Thomas, N. G. White, Chem. Soc. Rev. 2019, 48, 2596; For selected examples: b) A. Shokri, J. Schmidt, X.-B. Wang, S. R. Kass, J. Am. Chem. Soc. 2012, 134, 2094; c) A. Shokri, A. Abedin, A. Fattahi, S. R. Kass, J. Am. Chem. Soc. 2012, 134, 10646; d) A. Shokri, Y. Wang, G. A. O’Doherty, X.-B. Wang, S. R. Kass, J. Am. Chem. Soc. 2013, 135, 17919; e) A. Shokri, X.-B. Wang, Y. Wang, G. A. O’Doherty, S. R. Kass, J. Phys. Chem. A 2016, 120, 1661.

[14] a) A. Shokri, X.-B. Wang, S. R. Kass, J. Am. Chem. Soc. 2013, 135, 9525; b) M. Samet, S. R. Kass, J. Org. Chem. 2015, 80, 7727.

[15] C. Sperandio, J. Rodriguez, A. Quintard, Chem. Sci. 2020, 11, 1629.

[16] See for example: a) M. Marigo, D. Fielenbach, A. Braunton, A. Kjaersgaard, K. A. Jørgensen, Angew. Chem. Int. Ed. 2005, 44, 3703; b) T. D. Beeson, D. W. C. MacMillan, J. Am. Chem. Soc. 2005, 127, 8826; c) D. Enders, M. R. M. Hüttl, Synlett 2005, 991; d) D. D. Steiner, N. Mase, C. F. Barbas III., Angew. Chem. Int. Ed. 2005, 44, 3706.

[17] Z. Wang, L. Cheng, Z. Kai, F. Wu, Z. Liu, M. Cai, Bioorg. Med. Chem. Lett. 2014, 24, 3869.

[18] For the most significant examples see: a) J. T. Welch, K. Seper, S. Eswarakrishnan, J. Samartino, J. Org. Chem. 1984, 49, 4720; b) J. Saadi, M. Akakura, H. Yamamoto, J. Am. Chem. Soc. 2011, 133, 14248 ; c) M. C. Walker, B. W. Thuronyi, L. K. 
Charkoudian, B. Lowry, C. Khosla, M. C. Y. Chang, Science 2013, 341, 1089; d) J. Saadi, H. Wennemers, Nat. Chem. 2016, 8, 276.

[19] a) A. Quintard, J. Rodriguez, ACS Catal. 2017, 7, 5513; b) J Rodriguez, A. Quintard, Chimia 2018, 72, 580.

[20] A. Quintard, C. Sperandio, J. Rodriguez, Org. Lett. 2018, 20 , 5274.

[21] C.-L. Shao, R. G. Linington, M. J. Balunas, A. Centeno, P. Boudreau, C. Zhang, N. Engene, C. Spadafora, T. S. Mutka, D. E. Kyle, L. Gerwick, C.-Y. Wang, W. H. Gerwick, J. Org. Chem. 2015, 80, 7849.

[22] For a review, see: a) R. Britton, B. Kang, Nat. Prod. Rep. 2013, 30, 227; b) B. Kang, J. Mowat, T. Pinter, R. Britton, Org. Lett. 2009, 11, 1717; c) T. Borg, J. Danielsson, P. Somfai, Chem. Commun. 2010, 46, 1281; d) S. D. Halperin, R. Britton, Org. Biomol. Chem. 2013, 11, 1702; e) M. Bergeron-Brlek, M. Meanwell, R. Britton, Nature Communications 2015, 6, 6903.

[23] N. Halland, A. Braunton, S. Bachmann, M. Marigo, K. A. Jørgensen, J. Am. Chem. Soc. 2004, 126, 4790.

[24] A. Ricucci, J. Rodriguez, A. Quintard, Eur. J. Org. Chem. 2018, 3697.

[25] a) O. O. Fadeyi, C. W. Lindsley, Org. Lett. 2009, 11, 943; b) S. Harper, M. Ferrara, B. Crescenzi, M. Pompei, M. C. Palumbi, J. M. DiMuzio, M. Donghi, F. Fiore, U. Koch, N. J. Liverton, S. Pesci, A. Petrocchi, M. Rowley, V. Summa, C. Gardelli, J. Med. Chem. 2009, 52, 4820; c) J. H. Jones, C. Appayee, S. E. Brenner-Moyer, Eur. J. Org. Chem. 2014, 5273; d) A. S. Altiti, S. Bachan, W. Alrowhani, D. R. Mootoo, Org. Biomol. Chem. 2015, 13, 10328.

[26] J. Merad, P. Borkar, F. Caijo, J.-M. Pons, J.-L. Parrain, O. Chuzel, C. Bressy, Angew. Chem. Int. Ed. 2017, 56, 16052.
[27] a) S. Bertelsen, P. Dinér, R. L. Johansen, K. A. Jørgensen, $J$ Am. Chem. Soc. 2007, 129, 1536; b) N. Røjkjær Andersen, S. G. Hansen, S. Bertelsen, K. A. Jørgensen, Adv. Synth. Catal. 2009, 351, 3193; c) A. Quintard, A. Alexakis, Chem. Commun. 2011, 47, 7212 .

[28] C. Sperandio, J. Rodriguez, A. Quintard, Eur. J. Org. Chem. 2020, 2493.

[29] T. Hashimoto, M. Tori, Y. Asakawa, Chem. Pharm. Bull. 1986, 34, 1846.

[30] a) K. S. Feldman, R. E. Simpson, Tetrahedron Lett. 1989, 30, 6985; b) M. Miyashita, M. Hoshino, A. Yoshikoshi, Chem. Lett. 1990, 791; c) Z. A. Fang, G. J. Clarkson, M. Wills, Tetrahedron Lett. 2013, 54, 6834; d) G. He, Y. Wang, C. Lai, W. Li, R. Hong, Sci. China Chem. 2016, 59, 1197.

[31] C. Sperandio, G. Quintard, J.-V. Naubron, M. Giorgi, M. Yemloul, J.-L. Parrain, J. Rodriguez, A. Quintard, Chem. Eur. J. 2019, 25, 15098.

[32] a) A. R. Hirst, B. Escuder, J. F. Miravet, D. K. Smith, Angew Chem. Int. Ed. 2008, 47, 8002; b) D. Diaz Diaz, D. Kühbeck, R. J. Koopmans, Chem. Soc. Rev. 2011, 40, 427; c) L. E. Buerkle, S. J. Rowan, Chem. Soc. Rev. 2012, 41, 6089; d) C. D. Jones, J. W. Steed, Chem. Soc. Rev. 2016, 45, 6546; e) B. O. Okesola, D. K. Smith, Chem. Soc. Rev. 2016, 45, 4226; f) W. Fang, Y. Zhang, J. Wu, C. Liu, H. Zhu, T. Tu, Chem. Asian J. 2018, 13, 712 .

[33] C. Sperandio, J. Rodriguez, A. Quintard, Org. Biomol. Chem. 2020, 18, 2637. 